\title{
Peristaltic transport due to finite amplitude bending and contraction waves
}

\author{
By DENNIS E. WILSON \\ Department of Mechanical Engineering, University of South Carolina, Columbia \\ AND RONALD L. PANTON \\ Department of Mechanical Engineering, University of Texas, Austin
}

(Received 25 August 1977 and in revised form 19 May 1978)

This analysis demonstrates theoretically that a lateral bending wave propagating along the walls of a two-dimensional channel filled with a viscous incompressible fluid will induce a mean flow. In addition to this 'pure' bending wave, another possible condition is investigated: that of the superposition of an area contraction wave propagating with the same speed as the lateral bending wave. This second condition, called complex wave motion, takes into account the slight occlusion which occurs naturally at the amplitude peaks when a finite amplitude bending wave propagates along the walls of a container. A perturbation solution is found which satisfies NavierStokes equations for the case in which wave amplitude/wavelength is 'small'. However the wave amplitude is finite, in the sense that it is of the same order as the channel width. Under these conditions, the occlusion at the amplitude peaks is allowed to be of the same order as the channel width. For the case of a pure bending wave the motion induced by the peristaltis is found to be of second order in the pertusbation parameter, whereas in the more realistic case a first-order pumping effect is obtained.

\section{Introduction}

A travelling lateral bending wave imposed on the boundary of a container filled with a fluid induces a mean flow. The mechanism responsible for this effect is the same for either the classical case of the progressive area contraction-expansion wave or the pure lateral bending wave. This mechanism, being an acoustic or nonlinear streaming, is produced by the interaction of the convective acceleration terms.

The mechanisms responsible for peristaltic transport have been analysed only recently. One of the first publications was by Burns \& Parkes (1967). Over the ensuing five years approximately twenty papers dealt with the subject under various resstrictions on the Reynolds number, wavelength and amplitude ratio. Jaffrin \& Shapiro (1973) present a summary of these papers, organized according to geometry, fluid properties and the perturbation parameter. The perturbation parameter generally involves some combination of the wave amplitude, wavelength or transverse dimension of the container, either the thickness for a two-dimensional channel or the diameter for an axisymmetric tube. The results for the oscillating and mean flow components in the case of two-dimensional (Fung \& Yih 1968) and axisymmetric (Yin \& Fung 1969) geometry are similar. The only difference appears in the shape of the velocity 
profiles, much as with plane and cylindrical Poiseuille flow. Unfortunately, the analysis of a lateral bending wave imposed upon the boundary of a tube does not enjoy the luxury of the axisymmetric assumption. For this case a full three-dimensional analysis with highly complex boundary conditions is required. An integral control volume analysis (Wilson 1976) involving an arbitrary cross-sectional geometry demonstrated the same basic pumping phenomenon; consequently, an idealized two-dimensional model is employed in this investigation.

The only investigation found in the literature (Hanin 1968) for a lateral bending wave imposed on the walls of a two-dimensional channel concluded that no mean flow exists. Hanin's conditions were more restrictive than those in the present investigation in that he additionally considered the wave amplitude to be small compared with the channel thickness. Furthermore, equations of boundary-layer type were solved, further restricting the solution to low values of the reduced frequency, defined by (wave amplitude)(frequency)/(axial fluid velocity). In this analysis, the wave amplitude is of the same order as the channel thickness and no restrictions are initially placed on the transverse pressure gradient.

When a lateral bending wave of finite amplitude propagates along the length of a tube, two possible conditions arise concerning the cross-sectional geometry. First, the cross-sections can be assumed to move as rigid bodies without an accompanying area change. This is referred to as a pure bending wave and is treated in the first part of the analysis. Secondly, and perhaps more realistically, at the amplitude peaks, where the curvature is greatest, there is a tendency for the tube to occlude slightly owing to local deformation of the cross-sections. This condition, called complex wave motion, is also investigated and a substantial improvement in the pumping efficiency is found.

\section{Formulation of the in-phase wave problem}

The problem under consideration is a two-dimensional channel of uniform thickness $2 d$ filled with a homogeneous, viscous, incompressible fluid. The walls are flexible, allowing the imposition of travelling sinusoidal waves of amplitude $a$ and wavelength $\lambda$. Furthermore, the physical dimensions are restricted such that $a / \lambda=\epsilon$ and $a / d=O(1)$.

As might be expected from the geometry, shown in figure 1, a solution in the Cartesian co-ordinate system is rather complicated. Because of this difficulty the problem is formulated in the curvilinear co-ordinate system described below.

Three co-ordinate systems are shown for the model in figure 1. First, the $X, Y, Z$ system is fixed in space and the travelling wave is represented in this system by $H(X, t)$. Next, the $x, y, z$ system is 'attached' to the wave, i.e. it moves to the right with the wave speed $c$. Thus for this system the centre-line displacement is $h(x)$. Finally, the $\zeta, \eta, z$ system is a curvilinear orthogonal system which is fixed with respect to the $x, y, z$ system, hence it also moves to the right with the wave speed $c$. In this 'natural co-ordinate system', constant- $\eta$ lines coincide with the physical boundaries, at least to second order in terms of the perturbation parameter $\epsilon$.

The appropriate equations of motion for the fluid are to be formulated and solved in the natural co-ordinate system. The initial problem is to determine a co-ordinate transformation from the Cartesian system to the natural system. 


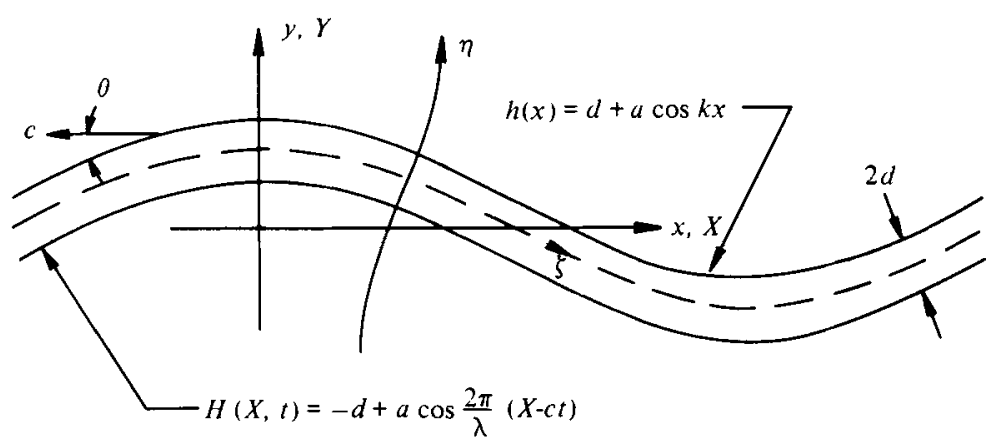

Frgure 1. Co-ordinate system for pure bending wave.

\subsection{Co-ordinate transformation}

If we define a co-ordinate transformation from the Cartesian to the natural system that is conformal, we can take advantage of various properties of analytic functions to simplify dramatically the resulting equations. This can be accomplished by constructing the natural co-ordinates from the streamlines and potential lines, which automatically satisfy the Cauchy-Riemann conditions. We need first to solve the complementary inviscid irrotational problem. This result will be used to construct a co-ordinate transformation which provides a convenient system in which to investigate the viscous rotational problem.

Since the potential flow problem is straightforward, only the perturbation solution for the potential and stream functions is given:

$$
\begin{aligned}
\Phi(X, Y, t) & =a c \sin (k X-\omega t) \sinh k Y / \cosh k d, \\
\Psi(X, Y, t) & =a c \cos (k X-\omega t) \cosh k Y / \cosh k d .
\end{aligned}
$$

By applying a simple Galilean transformation, we can write the potential lines and streamlines in the $x, y, z$ system as

$$
\begin{gathered}
\phi(x, y)=-c x+a c \sin k x \sinh k y / \cosh k d, \\
\psi(x, y)=-c y+a c \cos k x \cosh k y / \cosh k d .
\end{gathered}
$$

Immediately we can write down the following transformation relations:

$$
\begin{aligned}
& \zeta(x, y)=x-a \frac{\sinh k y}{\cosh k d} \sin k x, \\
& \eta(x, y)=y-a \frac{\cosh k y}{\cosh k d} \cos k x .
\end{aligned}
$$

These transformations are first-order accurate as a result of the approximations in the boundary conditions used in deriving (2.1) and (2.2). The approximation subject to $a / \lambda=\epsilon$ was that $\partial \Phi / \partial Y=\partial H / \partial t$ was evaluated at the mean boundary displacement. However, (2.5) and (2.6) can be considered exact for the displacement of the centre-line of the channel, and first-order accurate in general. We are interested in a solution to the internal flow bounded by parallel planes equidistant on either side of this line. It can be shown that in this restricted solution domain the transformations are in effect second-order accurate. This result can be seen from the non-dimensional 
transformations (2.22) and (2.23), and is due to the fact that we restrict the physical problem such that $d / \lambda=O(\epsilon)$. Consequently, we can obtain second-order solutions with these transformations and, furthermore, they are conformal.

\subsection{Transformation of the vorticity equation}

For plane two-dimensional flow, the vorticity has only a component in the $z$ direction. The resulting equation in the $x, y, z$ system is

$$
D \omega / D t=\nu \nabla^{2} \omega
$$

where

$$
\omega=\partial^{2} \psi / \partial x^{2}+\partial^{2} \psi / \partial y^{2}
$$

The $z$ co-ordinate is the same in both the Cartesian and the natural co-ordinate system, and since $\omega$ is a scalar quantity, it is also identical in both systems. We can thus write

$$
\omega(x, y)=\tilde{\omega}(\zeta, \eta)=J\left\{\partial^{2} \tilde{\psi} / \partial \zeta^{2}+\partial^{2} \tilde{\psi} / \partial \eta^{2}\right\}=J \tilde{\nabla}^{2} \tilde{\psi}
$$

where (2.9) takes advantage of the conformal properties of the mapping. Quantities with a tilde are functions of the $\zeta, \eta, z$ system and $J$ is the Jacobian of the transformation. Substituting (2.9) into the vorticity equation, exploiting the fact that $J$ is analytic, which implies $\nabla^{2} \omega=J^{2} \tilde{\nabla}^{4} \psi$ and $\partial J / \partial x=\partial J / \partial y=d J\left(z^{\prime}\right) / d z^{\prime}$, and adopting the notation $z^{\prime}=x+i y$ and $w=\zeta+i \eta$, we get after lengthy operations

$$
\frac{\partial}{\partial t} \tilde{\nabla}^{2} \tilde{\psi}+J\left\{\frac{\partial \tilde{\psi}}{\partial \eta} \tilde{\nabla}^{2} \frac{\partial \tilde{\psi}}{\partial \zeta}-\frac{\partial \tilde{\psi}}{\partial \zeta} \tilde{\nabla}^{2} \frac{\partial \tilde{\psi}}{\partial \eta}-\nu^{2} \tilde{\nabla}^{4} \tilde{\psi}\right\}=\tilde{\nabla}^{2} \tilde{\psi}\left(\frac{\partial \tilde{\psi}}{\partial \zeta}-\frac{\partial \tilde{\psi}}{\partial \eta}\right) \frac{d J^{-1}}{d w}
$$

Notice that for the identity mapping, $J=1$, the right-hand side equals zero, $\eta \rightarrow y$, $\zeta \rightarrow x$ and the result is the classical stream-function equation in Cartesian co-ordinates. The notation $\tilde{\nabla}^{2} \psi$ is used to denote $\operatorname{div}(\operatorname{grad}) \psi$ in the $\zeta, \eta$ system.

\subsection{Boundary conditions}

The boundary conditions that must be satisfied by the fluid on the walls are the no-slip and impermeability conditions. The approximate no-slip boundary conditions on a moving surface can be developed for a flexible but inextensible or flexible but extensible surface. Taylor (1951) has given explicitly the boundary conditions for a flexible but inextensible plate for small values of $a / \lambda$. We shall, however, adopt the simpler and perhaps more realistic condition of a flexible but extensible surface with a travelling wave, and assume only transverse displacements. This implies that $u=0$ and $v=\partial H / \partial t$ at $Y= \pm d+H$. In the $x, y, z$ system 'attached' to the travelling wave, the boundary conditions are simply

$$
u=-c, \quad v=0 \quad \text { at } \quad y= \pm d+h .
$$

In the $\zeta, \eta, z$ system these become

$$
\left.\begin{array}{l}
u=\zeta_{y} \partial \tilde{\psi} / \partial \zeta+\eta_{y} \partial \tilde{\psi} / \partial \eta=-c \\
v=-\zeta_{x} \partial \tilde{\psi} / \partial \zeta-\eta_{x} \partial \tilde{\psi} / \partial \eta=0
\end{array}\right\} \quad \text { at } \quad y= \pm d+h
$$

Using the Cauchy-Riemann conditions, $\zeta_{x}=\eta_{y}$ and $\zeta_{y}=-\eta_{x}$, and noting that $J \equiv \zeta_{x}^{2}+\zeta_{y}^{2}$, these become

$$
J \partial \psi / \partial \eta=-c \zeta_{x}, \quad J \partial \tau / \partial \zeta=-c \zeta_{y}
$$


Noting from the appendix that $\tilde{u}(\zeta, \eta)=J^{\frac{1}{2}} \partial \psi / \partial \eta$ and $\tilde{v}(\zeta, \eta)=-J \frac{1}{2} \partial \tilde{\psi} / \partial \zeta$, the boundary conditions for the two orthogonal velocity components become

$$
\left.\tilde{u}(\zeta, \eta)\right|_{\partial S}=-c \zeta_{x} J^{-\frac{1}{2}},\left.\quad \tilde{v}(\zeta, \eta)\right|_{\partial S}=c \zeta_{y} J^{-\frac{1}{2}}
$$

where the subscript $\partial S$ denotes that the function is to be evaluated at the surface, which is as yet unspecified in this system. Once the variables have been properly non-dimensionalized we shall return to $(2.17)$ and $(2.18)$ to define these boundary conditions in terms of $\eta$.

\subsection{Non-dimensionalization}

The ultimate solution to the problem, which is modelled approximately owing to the transformations, depends upon use of the correct non-dimensionalizing process. Otherwise, when the perturbation analysis is performed in the conformal plane the mathematical model will not conform to the physical problem. Therefore it is necessary to use different characteristic lengths in two co-ordinate directions. Let $\lambda$ be the characteristic length in the $\zeta$ direction and $d$ that in the $\eta$ direction. This process is similar to that for the long-wavelength shallow-water wave solutions given by Stoker (1957) and that in an analysis of the long-wave approximation by Zien \& Ostrach (1970). In effect, the scaling stretches the transverse co-ordinate suck that the solution domain in the conformal plane maintains the proper order when $\epsilon \rightarrow 0$. Otherwise the boundaries of the physical problem will collapse into a single line when mapped into the conformal plane. With these considerations, we introduce the following non-dimensional variables and parameters:

$$
\left.\begin{array}{cccc}
\bar{\zeta}=\zeta / \lambda, & \bar{x}=x / \lambda, & \bar{\eta}=\eta / d, & \bar{y}=y / d, \\
\bar{\psi}=\tilde{\psi} / c d, & \bar{u}=\tilde{u} / c, & \bar{v}=\tilde{v} \beta / c \epsilon, & \bar{t}=c t / \lambda, \\
\alpha=2 \pi d / \lambda, & \beta=a / d, & \epsilon=a / \lambda, & R e=c d / \nu .
\end{array}\right\}
$$

Notice that $u$ and $v$ are non-dimensionalized in a different manner. This scaling, like the $R e^{-\frac{1}{2}}$ scaling in the boundary-layer equations, satisfies the continuity equation.

Using these variables, the non-dimensional conformal transformation becomes

$$
\begin{aligned}
& \bar{\zeta}=\bar{x}-\epsilon \frac{\sinh \alpha \bar{y}}{\cosh \alpha} \sin 2 \pi \bar{x}, \\
& \bar{\eta}=\bar{y}-\beta \frac{\cosh \alpha \bar{y}}{\cosh \alpha} \cos 2 \pi \bar{x} .
\end{aligned}
$$

We can now determine the first-order-accurate inverse transformations by expanding $\sinh \alpha \bar{y}$ and $\cosh \alpha \bar{y}$ and solving. These become

$$
\bar{x}=\bar{\zeta}+O\left(\epsilon^{2}\right), \quad \bar{y}=\bar{\eta}+\beta \cos 2 \pi \bar{\zeta}+O\left(\epsilon^{2}\right) .
$$

The Jacobian can then be written as

$$
J=1-\epsilon\left(4 \pi \frac{\sinh \alpha \bar{y}}{\cosh \alpha} \cos 2 \pi \bar{x}\right)-\epsilon^{2}\left(4 \pi^{2} \frac{\cosh ^{2} \alpha \bar{y}}{\cosh ^{2} \alpha} \sin ^{2} 2 \pi \bar{x}\right)+O\left(\epsilon^{4}\right)
$$

By expanding in a Taylor series and noting that $\alpha=\epsilon 2 \pi / \beta$, it can be seen that $\max (\sinh \alpha \bar{y})=O(\epsilon)$. Thus we have the convenient result that $J(z)=1+O\left(\epsilon^{2}\right)$. The fact that the Jacobian is equal to one up to second order has important implications 
in the perturbation procedure and will be exploited several times in the analysis. It is also easy to show that $J_{(}(w)=1+O\left(\epsilon^{2}\right)$, where

$$
w(z)=\zeta+i \eta=z-i \frac{a}{\cosh k d} \cos k z
$$

Using these results, the stream-function equation can be written in non-dimensional form as

$$
\frac{\partial}{\partial t} \bar{\nabla}^{2} \psi+J\left\{\frac{\partial \bar{\psi}}{\partial \bar{\eta}} \bar{\nabla}^{2} \frac{\partial \bar{\psi}}{\partial \bar{\zeta}}-\frac{\partial \bar{\psi}}{\partial \bar{\zeta}} \bar{\nabla}^{2} \frac{\partial \bar{\psi}}{\partial \bar{\eta}}-\frac{\beta}{\epsilon R e} \bar{\nabla}^{4} \bar{\psi}\right\}=\bar{\nabla}^{2} \bar{\psi}\left(\frac{\partial \bar{\psi}}{\partial \bar{\eta}}-\frac{\epsilon}{\beta} \frac{\partial \bar{\psi}}{\partial \bar{\zeta}}\right) \epsilon^{2}
$$

where the modified 'Laplacian' operator is given by

and

$$
\bar{\nabla}^{2} \equiv \frac{\epsilon^{2}}{\beta^{2}} \frac{\partial^{2}}{\partial \bar{\zeta}^{2}}+\frac{\partial^{2}}{\partial \bar{\eta}^{2}}
$$

$$
\bar{\nabla}^{4} \equiv \frac{\epsilon^{4}}{\beta^{4}} \frac{\partial^{4}}{\partial \bar{\zeta}^{4}}+\frac{\epsilon^{2}}{\beta^{2}} \frac{\partial^{4}}{\partial \bar{\zeta}^{2} \partial \bar{\eta}^{2}}+\frac{\partial^{4}}{\partial \bar{\eta}^{4}}
$$

The boundary conditions can now be written as

$$
\left.\bar{u}\right|_{\partial S}=-\bar{\zeta}_{\bar{x}} J^{-\frac{1}{2}},\left.\quad \bar{v}\right|_{\partial S}=(\beta / \epsilon) \bar{\zeta}_{\bar{y}} J^{-\frac{1}{2}}
$$

and must be evaluated at $\bar{y}= \pm 1+\beta \cos 2 \pi \bar{\zeta}$. Combining this with (2.23) we find that the surface is given by $\bar{\eta}= \pm 1+O\left(\epsilon^{2}\right)$, and the boundary conditions become

$$
\bar{u}=-\bar{\zeta}_{\bar{x}} J^{-\frac{1}{2}}, \quad \bar{v}=(\beta / \epsilon) J^{-\frac{1}{2}} \quad \text { at } \quad \bar{\eta}= \pm 1+\epsilon^{2} \bar{f}(\bar{\zeta}, \bar{\eta}) .
$$

The function $\bar{f}(\bar{\zeta}, \bar{\eta})$ is lengthy and is left unspecified since it will not enter the boundary conditions up to second order.

\section{Method of solution of pure bending wave problem}

Using the long-wavelength approximation, the stream function can be expanded in an asymptotic series as

$$
\bar{\psi}(\bar{\zeta}, \bar{\eta}, \bar{t})=\bar{\psi}_{0}(\bar{\zeta}, \bar{\eta}, \bar{t})+\epsilon \bar{\psi}_{1}(\bar{\zeta}, \bar{\eta}, \bar{t})+\epsilon^{2} \bar{\psi}_{2}(\bar{\zeta}, \bar{\eta}, \bar{t})+O\left(\epsilon^{3}\right) .
$$

Substituting this expansion into (2.26) and following the usual ordering procedure in straightforward perturbation problems results in the following equations for the first three orders in $\epsilon$ :

$$
\partial^{4} \bar{\psi}_{0} / \partial \bar{\eta}^{4}=0
$$

$$
\frac{\beta}{R e} \frac{\partial^{4} \bar{\psi}_{1}}{\partial \bar{\eta}^{4}}=\frac{\partial^{3} \bar{\psi}_{0}}{\partial \bar{t}_{\partial} \partial \bar{\eta}^{2}}-\frac{\partial \bar{\psi}_{0}}{\partial \bar{\zeta}} \frac{\partial^{3} \bar{\psi}_{0}}{\partial \bar{\eta}^{3}}+\frac{\partial \bar{\psi}_{0}}{\partial \bar{\eta}^{2}} \frac{\partial^{3} \bar{\psi}_{0}}{\partial \bar{\zeta} \partial \bar{\eta}^{2}},
$$

$$
\frac{\beta}{R e} \frac{\partial^{1} \bar{\psi}_{2}}{\partial \bar{\eta}^{4}}=-\frac{\beta}{R e} \frac{\partial^{1} \bar{\psi}_{0}}{\partial \bar{\eta}^{2} \partial \bar{\zeta}^{2}}+\frac{\partial^{3} \bar{\psi}_{1}}{\partial \bar{t} \partial \bar{\eta}^{2}}+\frac{\partial \bar{\psi}_{1}}{\partial \bar{\eta}} \frac{\partial^{3} \bar{\psi}_{0}}{\partial \bar{\zeta}_{\partial} \partial \bar{\eta}^{2}}+\frac{\partial \bar{\psi}_{0}}{\partial \bar{\eta}} \frac{\partial^{3} \bar{\psi}_{1}}{\partial \bar{\zeta}_{\partial} \bar{\eta}^{2}}-\frac{\partial \bar{\psi}_{0}}{\partial \bar{\zeta}_{0}} \frac{\partial^{3} \bar{\psi}_{1}}{\partial \bar{\eta}^{3}}-\frac{\partial \bar{\psi}_{1}}{\partial \bar{\zeta}^{3}} \frac{\partial^{3} \bar{\psi}_{0}}{\partial \bar{\eta}^{3}}
$$

To complete the solution it is necessary to develop the appropriate set of equations relating the 'axial' pressure gradient to the stream function. This is done by writing the $x$ and $y$ momentum equation in terms of the stream function and using the transformation relations. The $x$ and $y$ momentum equations are multiplied by $\zeta_{x}$ and $\zeta_{y}$ respectively and then added. The non-dimensional axial pressure gradient is defined by

$$
P \equiv \frac{\partial p / \partial \zeta}{\rho c^{2} / d}
$$


Defining the transverse pressure gradient similarly, then expanding $P$ in an asymptotic series, the following relations up to second order are obtained:

$$
\begin{gathered}
P_{0}=\frac{1}{R e} \frac{\partial^{3} \bar{\psi}_{0}}{\partial \bar{\eta}^{3}}, \\
P_{1}=\frac{1}{R e} \frac{\partial^{3} \bar{\psi}_{1}}{\partial \bar{\eta}^{3}}-\frac{\partial^{3} \bar{\psi}_{0}}{\partial \bar{t} \partial \bar{\eta}}-\frac{\partial \bar{\psi}_{0}}{\partial \bar{\eta}} \frac{\partial^{2} \bar{\psi}_{0}}{\partial \bar{\eta} \partial \bar{\zeta}}+\frac{\partial \bar{\psi}_{0}}{\partial \bar{\zeta}} \frac{\partial^{2} \bar{\psi}_{0}}{\partial \bar{\eta}^{2}} \\
P_{2}=\frac{1}{R e}\left\{\frac{\partial^{3} \bar{\psi}_{2}}{\partial \bar{\eta}^{3}}+\frac{\partial^{3} \bar{\psi}_{0}}{\partial \bar{\zeta}^{2} \partial \bar{\eta}}\right\}+\frac{\partial \bar{\psi}_{1}}{\partial \bar{\zeta}} \frac{\partial^{2} \bar{\psi}_{0}}{\partial \bar{\eta}^{2}}-\frac{\partial \bar{\psi}_{0}}{\partial \bar{\eta}} \frac{\partial^{2} \bar{\psi}_{1}}{\partial \bar{\eta} \partial \bar{\zeta}}+\frac{\partial \bar{\psi}_{0}}{\partial \bar{\zeta}^{2}} \frac{\partial^{2} \bar{\psi}_{1}}{\partial \bar{\eta}^{2}}-\frac{\partial \bar{\psi}_{1}}{\partial \bar{\eta}} \frac{\partial^{2} \bar{\psi}_{0}}{\partial \bar{\zeta}^{2}}+\frac{\partial^{2} \bar{\psi}_{1}}{\partial \bar{t} \partial \bar{\eta}} .
\end{gathered}
$$

All that remains now is to specify the boundary conditions up to $O\left(\epsilon^{2}\right)$. Since $\epsilon$ appears both implicitly and explicitly, we first expand the boundary conditions about $\eta= \pm 1$ in a Taylor series. Next, expanding $\bar{u}$ and $\bar{v}$ in an asymptotic series similar to (3.1), the boundary conditions become

$$
\left.\begin{array}{rl}
\bar{u}_{0}+\epsilon \bar{u}_{1}+\epsilon^{2} \bar{u}_{2}+O\left(\epsilon^{3}\right) & =-\bar{\zeta}_{\bar{x}} J^{-\frac{1}{2}} \\
\bar{v}_{0}+\epsilon \bar{v}_{1}+\epsilon^{2} \bar{v}_{2}+O\left(\epsilon^{3}\right) & =(\beta / \epsilon) \bar{\zeta}_{\bar{y}} J^{-\frac{1}{2}}
\end{array}\right\} \quad \text { at } \quad \eta= \pm 1 .
$$

By expanding sinh $\alpha \bar{y}$ and $\cosh \alpha \bar{y}$ in the expression for $J$ given by (2.24) then using the inverse transformation relations given by $(2.22)$ and $(2.23)$, we find that

$$
J=1-\epsilon^{2}\left\{\left(8 \pi^{2} / \beta\right) \bar{\eta} \cos 2 \pi \bar{\zeta}+8 \pi^{2} \cos ^{2} 2 \pi \bar{\zeta}-4 \pi^{2} \sin ^{2} 2 \pi \bar{\zeta}\right\}+O\left(\epsilon^{3}\right)
$$

Similarly $\bar{\zeta}_{\bar{x}}$ and $\bar{\zeta}_{\bar{y}}$ become

$$
\begin{aligned}
& \bar{\zeta}_{\bar{x}}=1-\epsilon^{2}\left\{\left(4 \pi^{2} / \beta\right) \bar{\eta} \cos 2 \pi \bar{\zeta}+4 \pi^{2} \cos ^{2} 2 \pi \bar{\zeta}\right\}+O\left(\epsilon^{3}\right) \\
& \bar{\zeta}_{\bar{y}}=\epsilon^{2}(2 \pi / \beta) \sin 2 \pi \bar{\zeta}+O\left(\epsilon^{3}\right)
\end{aligned}
$$

Substituting these three equations into the right-hand side of (3.8) and (3.9), expanding and collecting like powers of $\epsilon$, the boundary conditions become

$$
\begin{aligned}
& \bar{u}_{0}( \pm 1)=-1, \quad \bar{v}_{0}( \pm 1)=0, \\
& \bar{u}_{1}( \pm 1)=0, \quad \bar{v}_{1}( \pm 1)=2 \pi \sin 2 \pi \bar{\zeta}, \\
& \bar{u}_{2}( \pm 1)=2 \pi^{2} \sin ^{2} 2 \pi \bar{\zeta}, \quad \bar{v}_{2}( \pm 1)=0 .
\end{aligned}
$$

It is interesting that the same equations can be obtained in a much less rigorous manner, by referring to figure 1 and writing

$$
\left.\tilde{u}\right|_{\partial S}=-c \cos \theta,\left.\quad \tilde{v}\right|_{\partial S}=c \sin \theta,
$$

where $\theta=d h / d x=-a k \sin k x$. Substituting for $\theta$, we then have

$$
\left.\tilde{u}\right|_{\partial S}=-c \cos (-a k \sin k x),\left.\quad \tilde{v}\right|_{\partial S}=c \sin (-a k \sin k x) .
$$

Non-dimensionalizing and expanding the sines and cosines in Taylor series gives

$$
\begin{aligned}
& \bar{u}( \pm 1)=-1-\epsilon^{2}\left(2 \pi \sin ^{2} 2 \pi \bar{\zeta}\right)+O\left(\epsilon^{4}\right), \\
& \bar{v}( \pm 1)=\epsilon(2 \pi \sin 2 \pi \bar{\zeta})+O\left(\epsilon^{3}\right) .
\end{aligned}
$$

Then by expanding $\bar{u}$ and $\bar{v}$ in an asymptotic series in $\epsilon$, the same boundary conditions are obtained. Although less rigorous, this approach provides more physical insight as to the origin of the terms in the perturbation boundary conditions. 
The system now contains differential equations in terms of the stream function and boundary conditions in terms of velocity components. This is a somewhat unusual formulation; however, the boundary conditions are more compact and physically instructive when specified in terms of velocities for a natural orthogonal curvilinear co-ordinate system such as the $\zeta, \eta, z$ system. We shall thus need a relation between the velocity components and the stream function. By using (A 1) and (A 2) in the appendix and expanding both the stream function and the velocity components as before, we obtain

$$
\begin{aligned}
\bar{u}_{0}= & \partial \bar{\psi}_{0} / \partial \bar{\eta}, \quad \bar{v}_{0}=-\partial \bar{\psi}_{0} / \partial \bar{\zeta} \\
\bar{u}_{1}= & \partial \bar{\psi}_{1} / \partial \bar{\eta}, \quad \bar{v}_{1}=-\partial \bar{\psi}_{1} / \partial \bar{\zeta}, \\
\bar{u}_{2}= & \partial \bar{\psi}_{2} / \partial \bar{\eta}+g \partial \bar{\psi}_{0} / \partial \bar{\eta}, \quad \bar{v}_{2}=-\partial \bar{\psi}_{2} / \partial \bar{\zeta}-g \partial \bar{\psi}_{0} / \partial \bar{\zeta} \\
& g=2 \pi^{2} \sin ^{2} 2 \pi \bar{\zeta}-4 \pi^{2} \cos ^{2} 2 \pi \bar{\zeta}-\left(4 \pi^{2} / \beta\right) \bar{\eta} \cos 2 \pi \bar{\zeta} .
\end{aligned}
$$

where

The second terms in the equations for $\bar{u}_{2}$ and $\bar{v}_{2}$ both come from the interaction of the curvature and the uniform translation at velocity $c$ of the $\zeta, \eta, z$ system even when no basic flow from an imposed pressure gradient is present. At first the expressions for $\bar{u}_{2}$ and $\vec{v}_{2}$ appear not to satisfy continuity up to $O\left(\epsilon^{2}\right)$, however this is not the case. In the natural orthogonal co-ordinate system continuity is expressed by

$$
\nabla \cdot \mathbf{v}=\frac{1}{\tilde{J}}\left[\frac{\partial}{\partial \zeta}\left(\tilde{u} \tilde{J}_{\frac{1}{2}}\right)+\frac{\partial}{\partial \eta}\left(\tilde{v} \tilde{J}_{\frac{1}{2}}\right)\right]
$$

Using $\tilde{u}$ and $\tilde{v}$ from the appendix, we see that continuity is indeed satisfied. Furthermore, by non-dimensionalizing the continuity equation, then expanding the velocities and the Jacobian in terms of $\epsilon$, it can be shown that $\bar{u}_{2}$ and $\bar{v}_{2}$ as given by (3.19) satisfy the $O\left(\epsilon^{2}\right)$ continuity equation.

Having now completed the formulation of the problem and developed the system of equations deseribing the flow up to second order, with appropriate boundary conditions, the solutions can now be obtained in a very straightforward manner.

The basic [i.e. $O(1)$ ] flow is described by (3.2), (3.13) and (3.17). The solution is found to be

$$
\partial \bar{\psi}_{0} / \partial \vec{\eta}=\bar{u}_{0}=k_{0}(t)\left[1-\bar{\eta}^{2}\right]-1 \text {. }
$$

Using (3.5) for the pressure gradient, we see that

$$
P_{0}=\frac{1}{R e} \frac{\partial^{3} \bar{\psi}_{0}}{\partial \bar{\eta}^{3}}=-\frac{2 k_{0}(t)}{R e}
$$

The pressure gradient is allowed to be a function of time and the solution is valid within the limits imposed by the non-dimensional quantities defined by (2.19). Denoting the period of the viscous wave as $d^{2} / \nu$ and the period of the travelling wave as $\lambda / c$, we require that

$$
\frac{d^{2} / \nu}{\lambda / c} \ll 1
$$

Referring to (2.19), this is equivalent to $(d / \lambda) R e \ll 1$. Since $R e=O(1)$ and $d / \lambda=O(\epsilon)$, this allows slowing varying, or quasi-steady pressure gradients. Thus (3.20) becomes

$$
\bar{u}_{0}=\frac{1}{2} \operatorname{Re} P_{0}\left(1-\bar{\eta}^{2}\right)-1 \text {. }
$$


The non-dimensional flow rate $\bar{Q}$ is given by

$$
\frac{1}{2} \bar{Q}_{0}=\left.\bar{\psi}_{0}\right|_{\eta=1}-\left.\bar{\psi}_{0}\right|_{\eta=0}=-\frac{1}{3} P_{0} R e-1 \text {. }
$$

For $P_{0}=0$, which corresponds to a zero imposed basic pressure gradient, the flow rate is $\bar{Q}=-2$ as expected. This is because the $\zeta, \eta, z$ system is translating to the right at non-dimensional velocity $\bar{u}=-1$ and the non-dimensional channel width is $\bar{d}=2$.

The first-order flow is described by (3.3) (where the right-hand side is zero for $\left.P_{0}=0\right),(3.14)$ and (3.18). Again the solution is trivial and the result is

$$
\bar{\psi}_{1}(\bar{\zeta}, \bar{\eta}, \bar{t})=k_{1}(\bar{t})\left[\bar{\eta}-\frac{1}{3} \bar{\eta}^{3}\right]-\cos 2 \pi \bar{\zeta}+c_{1}(\bar{t}) .
$$

The constant $c_{1}(\bar{t})$ is arbitrary, since it does not affect the solution for the velocity components, and is set equal to zero. Using (3.6), the relation for $k_{1}(t)$ is seen to be

yielding

$$
\begin{gathered}
P_{1}=-(2 / R e) k_{1}(t), \\
\bar{\psi}_{1}=-\frac{1}{2} P_{1} \operatorname{Re}\left(\bar{\eta}-\frac{1}{3} \bar{\eta}^{3}\right)-\cos 2 \pi \bar{\zeta} .
\end{gathered}
$$

The parameter $P_{1}$ corresponds to the pressure gradient due to unspecified end conditions and is not related to mass transport induced by the transverse travelling wave. Setting $P_{1}=0$ implies that $u_{1}(\bar{\zeta}, \bar{\eta})=0$ and $\bar{v}_{1}(\bar{\zeta}, \bar{\eta})=2 \pi \sin 2 \pi \bar{\zeta}$.

The equation for $\bar{u}_{1}$ might appear incorrect since we expect a purely periodic component as with classical peristaltic pumping. In effect, this is what exists with respect to the $X, Y, Z$ system. Referring to figure 1 , it is seen that constant- $\zeta$ planes deform periodically with period $2 \pi$. When observed in the $X, Y, Z$ system, or a reference frame fixed on the channel, we see a velocity which is periodic in $X$ and varies as $\sinh y$, which is consistent with a small amplitude solution by Wilson (1977).

The second-order solution is found by solving the system given by (3.4) for the stream function with (3.15) as the boundary conditions. Using the results for $\bar{\psi}_{0}$ and $\bar{\psi}_{1}$, this system becomes

$$
\begin{gathered}
\partial^{4} \bar{\psi}_{2} / \partial \bar{\eta}^{4}=0 \\
\bar{u}_{2}( \pm 1)=2 \pi^{2} \sin ^{2} 2 \pi \bar{\zeta}, \quad \bar{v}_{2}( \pm 1)=0
\end{gathered}
$$

The general solution for $\partial \bar{\psi}_{2} / \partial \bar{\eta}$ is

$$
\partial \bar{\psi}_{2} / \partial \bar{\eta}=h_{1}(\bar{\zeta}, \bar{t})+h_{2}(\bar{\zeta}, \bar{t}) \eta+h_{3}(\bar{\zeta}, \bar{t}) \bar{\eta}^{2} .
$$

Using (3.19) for $\bar{u}_{2}$ yields

$$
\begin{aligned}
\bar{u}_{2}(\bar{\zeta}, \bar{\eta}, \bar{t})=h_{1}(\bar{\zeta}, \bar{t})+h_{2}(\bar{\zeta}, \bar{t}) \eta+h_{3}(\bar{\zeta}, \bar{t}) \bar{\eta}^{2} \\
-\left(2 \pi^{2} \sin ^{2} 2 \pi \bar{\zeta}-4 \pi^{2} \cos ^{2} 2 \pi \bar{\zeta}-\left(4 \pi^{2} / \beta\right) \bar{\eta} \cos 2 \pi \bar{\zeta}\right) .
\end{aligned}
$$

Applying the boundary conditions, the result is

$$
\bar{u}_{2}(\bar{\zeta}, \bar{\eta}, \bar{t})=2 \pi^{2}\left(1-\bar{\eta}^{2}\right) \sin ^{2} 2 \pi \bar{\zeta}+k_{2}(\bar{t})\left(1-\bar{\eta}^{2}\right) .
$$

Applying (3.7) for the pressure gradient, this result can be rewritten as

$$
P_{2}=(-2 / R e)\left[\pi^{2}(1-\cos 4 \pi \bar{\zeta})+k_{2}(t)\right]
$$

For the second-order solution we see that the pressure gradient is composed of two parts. As before in the lower-order terms, $k_{2}$ is related to the external imposed pressure 


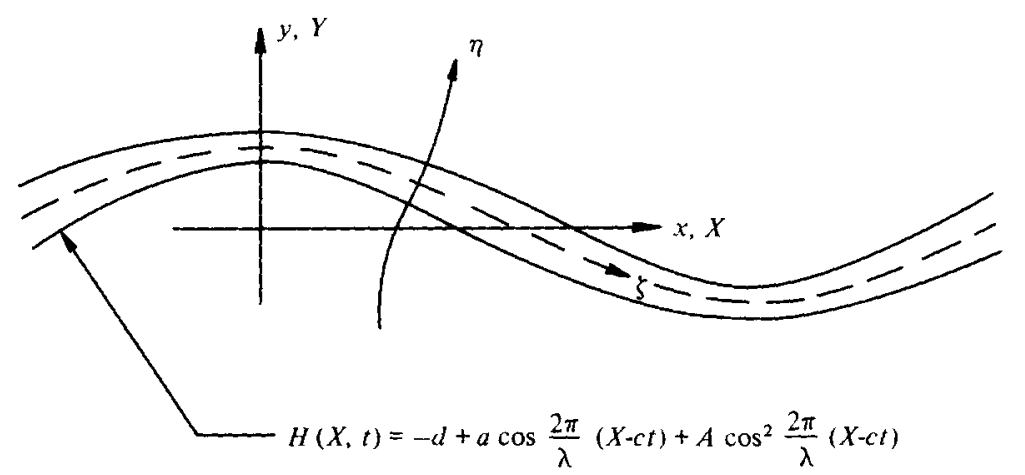

Figure 2. Co-ordinate system for complex wave (bending plus area contraction).

gradient. The $-2 \pi^{2} / R e$ contribution is due to the wall motion and produces a mean wall-induced flow $O\left(\epsilon^{2}\right)$. The average mean flow will be computed and discussed in the last section. At that stage we shall be able to contrast the results with the problem where a complex wall motion is allowed.

\section{Formulation of the complex wave problem}

The second part of the analysis involves a lateral bending wave with an accompanying area contraction proportional to the local curvature of the travelling wave. This occlusion or area contraction wave is a natural phenomenon resulting from local deformation and is a maximum at the amplitude peaks, zero midway between peaks and travels with the same wave speed as the bending wave.

Two equivalent approaches to the problem are possible. One consists of developing new co-ordinate transformations in which constant- $\eta$ surfaces coincide approximately with the boundaries of the system. The other approach, and the one followed here, uses the same co-ordinate transformations and specifies new boundary conditions. In this approach the boundary conditions are not applied on constant co-ordinate lines, which is usually undesirable. However, because of the simple nature of the differential equations, this produces no difficulties and yields the most direct results. The coordinate systems for the model are shown in figure 2 . The wall motion is now given by

$$
\hat{H}(X, t)= \pm d+a \cos \frac{2 \pi}{\lambda}(X-c t) \mp A \cos ^{2} \frac{2 \pi}{\lambda}(X-c t) .
$$

In the moving system we can specify this wall motion as

$$
\hat{h}(x)= \pm d+a \cos k x \mp A \cos ^{2} k x .
$$

We shall adopt the hat notation, e.g. $\hat{h}(x)$, for the variables that have common symbols for the pure bending and complex wave solutions.

The relevant co-ordinate transformations are given by (2.5) and (2.6). All that remains is to formulate the boundary conditions. The more direct approach, which began with (3.16), will be used with $\theta(x)$ now written as $\theta(x)$. The boundary conditions then become

$$
\left.\hat{u}\right|_{\partial S}=-c \cos \hat{\theta},\left.\quad \hat{v}\right|_{\partial S}=c \sin \hat{\theta},
$$

where

$$
\theta(x)=d \hat{h} / d x=-a k \sin k x \mp 2 A k \sin k x \cos k x \text {. }
$$


Substituting the expression for $\hat{\theta}(x)$ and using the non-dimensional variables defined by $(2.19)$ yields

$$
\begin{aligned}
& \hat{u}=-\cos \{-\epsilon 2 \pi \sin 2 \pi \bar{\zeta} \pm \epsilon \delta 4 \pi \sin 2 \pi \bar{\zeta} \cos 2 \pi \bar{\zeta}\} \\
& \hat{v}=\sin \{-\epsilon 2 \pi \sin 2 \pi \bar{\zeta} \pm \epsilon \delta 4 \pi \sin 2 \pi \bar{\zeta} \cos 2 \pi \bar{\zeta}\}
\end{aligned}
$$

applied at $\hat{\eta}= \pm 1 \mp \delta \beta \cos ^{2} 2 \pi \bar{\zeta}$, where $\delta=A / a=O(1)$.

Expanding $\hat{u}$ and $\hat{v}$ in an asymptotic series in $\epsilon$, expanding the right-hand sides of (4.6) and (4.7) in a Taylor series and collecting like powers of $\epsilon$ yields the following equations up to first order:

$$
\begin{aligned}
& \hat{u}_{0}( \pm N)=-1, \quad \hat{v}_{0}( \pm N)=0, \\
& \hat{u}_{1}( \pm N)=0, \quad \hat{v}_{1}( \pm N)=2 \pi \sin 2 \pi \bar{\zeta} \mp 4 \pi \sin 2 \pi \bar{\zeta} \cos 2 \pi \bar{\zeta}
\end{aligned}
$$

where for convenience $N \equiv 1-\delta \beta \cos ^{2} 2 \pi \bar{\zeta}$. Notice that the boundary conditions are essentially those given by (3.13) and (3.14). This is to be expected since as $\epsilon \rightarrow 0$ we require $\delta=O(1)$ and the basic flow is identical. However, the transverse wall velocity will differ in the first-order solution by an additional component $4 \pi \sin 2 \pi \bar{\zeta} \cos 2 \pi \bar{\zeta}$, which can be thought of as a second-harmonic wave, where $\sin 2 \pi \bar{\zeta}$ represents the firstharmonic wave.

\section{Method of solution of complex wave problem}

The solution technique is identical to that for pure bending wave motion. The transformations and relevant equations of motion are identical, the only difference being the new set of boundary conditions. The system of equations to be solved for the basic flow is

$$
\begin{gathered}
\partial^{4} \hat{\psi}_{0} / \partial \bar{\eta}^{4}=0, \\
\hat{u}_{0}( \pm N)=-1, \quad \hat{v}( \pm N)=0
\end{gathered}
$$

and the system for the first-order flow is

$$
\begin{gathered}
\frac{\beta}{R e} \frac{\partial^{4} \hat{\psi}_{1}}{\partial \bar{\eta}^{4}}=\frac{\partial^{3} \hat{\psi}_{0}}{\partial \bar{t} \partial \bar{\eta}^{2}}-\frac{\partial \hat{\psi}_{0}}{\partial \bar{\zeta}} \frac{\partial^{3} \hat{\psi}_{0}}{\partial \bar{\eta}^{3}}+\frac{\partial \hat{\psi}_{0}}{\partial \bar{\eta}} \frac{\partial^{3} \hat{\psi}_{0}}{\partial \bar{\zeta} \partial \bar{\eta}}, \\
\hat{u}_{1}( \pm N)=0, \quad \hat{v}_{1}( \pm N)=2 \pi \sin 2 \pi \bar{\zeta} \mp 4 \pi \sin 2 \pi \bar{\zeta} \cos 2 \pi \bar{\zeta}
\end{gathered}
$$

Solving the equation for the basic flow yields

$$
\hat{\psi}_{0}=\hat{k}_{0}(t)\left[\frac{\bar{\eta}}{N}-\frac{1}{3}\left(\frac{\eta}{N}\right)^{3}\right]-\bar{\eta}+\hat{c}_{0}(\bar{t})
$$

where $\hat{c}_{0}(t)$ is an arbitrary constant which is set equal to zero and $\hat{k}_{0}(t)=-\frac{1}{2} \operatorname{Re} P_{0}$. The equation for $u_{0}$ is

$$
\hat{u}_{0}=\frac{-P_{0} \operatorname{Re}}{2 N}\left[1-\left(\frac{\eta}{N}\right)^{2}\right]-1 \text {. }
$$

If $\delta$ is allowed to become zero then $N=1$ and the result is the same as for the first problem.

The solution to (4.12)-(4.14) for the first-order flow is found by integrating the stream-function equation and using the results for the basic flow; this yields

$$
\hat{\psi}_{1}=\hat{g}_{0}(\bar{\zeta}, \bar{t})+\hat{g}_{1}(\bar{\zeta}, \bar{t}) \bar{\eta}+g_{2}(\bar{\zeta}, \bar{t}) \bar{\eta}^{2}+g_{3}(\bar{\zeta}, \bar{t}) \bar{\eta}^{3}
$$


Applying the boundary conditions gives

$$
\hat{\psi}_{1}=\left[\frac{3}{2} \pi \cos ^{2} 2 \pi \bar{\zeta}+\hat{k}_{1}(\bar{t})\right]\left[\frac{\eta}{N}-\frac{1}{3}\left(\frac{\bar{\eta}}{N}\right)^{3}\right]-2 \pi \cos 2 \pi \bar{\zeta}+\hat{c}_{1}(\bar{t}) .
$$

As before, $c_{1}(\bar{t})=0$. Using the pressure-gradient relation given by (3.6) shows that $k_{1}(\bar{t})$ corresponds to the external imposed pressure gradient of first order, which is then assumed to be zero. Rewriting the $\cos ^{2} 2 \pi \bar{\zeta}$ term, (4.17) becomes

$$
\hat{\psi}_{1}=\frac{3 \pi}{4}[1+\cos 4 \pi \bar{\zeta}]\left[\frac{\bar{\eta}}{N}-\frac{1}{3}\left(\frac{\bar{\eta}}{N}\right)^{3}\right]-2 \pi \cos 2 \pi \bar{\zeta} .
$$

The axial velocity then can be written as

$$
\hat{u}_{1}=\frac{3 \pi}{4 N}[1+\cos 4 \pi \bar{\zeta}]\left[1-\left(\frac{\bar{\eta}}{N}\right)^{2}\right]
$$

where the pressure gradient is composed of a constant plus a periodic component given by

$$
P_{1}=\left(-3 \pi / 2 \operatorname{Re} N^{3}\right) \quad[1+\cos 4 \pi \bar{\zeta}] .
$$

This produces the interesting result that the pressure gradient induced by the wall motion and the resulting fluid transport is of first order in $a / \lambda$, implying essentially an order-of-magnitude improvement in pumping.

\section{Discussion and summary}

The problem of peristaltic fluid motion induced by both pure and complex bending waves has been analysed using a perturbation analysis in terms of the parameter $a / \lambda$. The results are in general agreement with those of a small amplitude analysis (Wilson 1977) where $a / d=\epsilon$, whereas in this problem $a / d=O(1)$. Specifically, the results demonstrate that the fluid transport is a second-order effect for a pure bending wave and that first-order effects exist for complex wave motion.

It is interesting to note that the mean flow is independent of $R e$ whereas for the small amplitude bending wave solution (Wilson 1977) it is a function of $R e^{2}$. This is due to the assumptions of boundary-layer type with respect to the scaling of the length and velocity employed in this investigation. The problem then becomes similar to the streaming which occurs in oscillating boundary layers, which was discussed by Lighthill (1953) and then by Lin (1956), where the mean flow is independent of $R e$ but the streaming effect is dependent on viscosity. That is, an inviscid solution yields purely periodic behaviour up to second order. This type of behaviour is also referred to as acoustic streaming (Schlichting 1968, p. 414).

Summarizing the complex wave solution, the interesting result of a first-order pumping was found to be induced by the wall motion for a fluid with no external pressure gradient. Noting that for this situation $A / a=O(1)$ and $a / d=O(1)$, we see that $A / d=O(1)$ and it is obvious that full occlusion of the channel is possible at the amplitude peaks of the lateral bending wave. Under this condition, a trapped bolus of fluid is simply transported downstream as the lateral bending wave propagates down the channel. Since the bending wave amplitude ratio $a / \lambda$ is of first order, the mean fluid transport will also be of first order in $a / \lambda$. 
The magnitude of the space- and time-averaged axial velocity, denoted as $|\langle u\rangle|$, can easily be obtained for both cases. Taking $k_{2}=0$ (i.e. no imposed pressure gradient up to second order), we find first for the pure bending wave that

$$
\left|\left\langle\bar{u}_{2}\right\rangle\right|=\frac{4}{3} \pi^{2}(a / \lambda)^{2} c .
$$

This can then be compared with the space- and time-averaged velocity for the complex wave:

$$
\left|\left\langle\hat{u}_{1}\right\rangle\right|=\frac{3}{4} \frac{\pi}{|N|} \frac{a}{\lambda} c
$$

where $|N|=1-\frac{1}{2} \delta \beta=1-\frac{1}{2} A / a$. For this case, as the occlusion increases, i.e. $|N| \rightarrow \frac{1}{2}$, we find

$$
\left|\left\langle u_{1}\right\rangle\right| \rightarrow \frac{3}{2} \pi(a / \lambda) c
$$

An expression can now be readily formulated for the effective pressure gradient generated by a lateral bending wave with an area contraction superimposed at the amplitude peaks. Recalling the definition of the non-dimensional pressure gradient $P_{1}$, the 'axial pressure gradient' becomes

$$
\frac{\partial p}{\partial \zeta}=\frac{\rho c^{2}}{d} \frac{3 \pi}{2 \operatorname{Re}{\overline{N^{3}}}^{3}}=\frac{3}{2} \pi\left(\frac{\mu c}{d^{2}}\right) \frac{1}{\bar{N}^{3}} .
$$

Defining the increase in pressure per wavelength as

$$
\Delta p_{\lambda}=\int_{0}^{\lambda}\left(\frac{\partial p}{\partial \zeta}\right) d \zeta
$$

we have

$$
\Delta p_{\lambda}=\frac{3}{2} \pi\left(\frac{\mu c}{d}\right) \frac{\beta}{\epsilon \bar{N}^{3}} .
$$

A major difference in this peristaltic-pumping analysis, in addition to the fact that the travelling-wave wall motion is quite different from the classical sinusoidal area contraction-expansion wave, is that a first-order pumping effect can exist. Furthermore, natural phenomena employing peristaltic pumping, such as occur in the human body, may use such first-order effects, as opposed to the usual second-order streaming. In this case complex wave forms other than the purely sinusoidal wave may exist. More probably, nonlinear interactions due to relaxation oscillations in the confining walls may exist. In fact, observations of blood vessels indicate that a rapid contraction wave followed by a slow expansion wave actually exists. Consequently, a more reasonable mathematical model should include this nonlinear frequency effect. Here the method followed in this paper could be extremely powerful, since any wave form can be assumed and the corresponding transformations obtained. Then the vorticity equation can be mapped into the natural co-ordinate system, where the solution should be straightforward. The effect of these nonlinear relaxation oscillations will be the subject of a future investigation. 


\section{Appendix}

It was necessary in the analysis to have relationships between the velocity components and the gradients of the stream function in the natural curvilinear co-ordinate system. These can be obtained in the following straightforward manner.

Let $f$ and $g$ denote the equations of the stream surfaces. Then for an incompressible flow the velocity is given by

$$
\mathrm{V}=\nabla f \times \nabla g
$$

The expression for the gradient in the natural co-ordinate system must be derived before proceeding. Consider a differential line $d s$ in the $\zeta, \eta$ plane:

$$
(d s)^{2}=h_{\eta}^{2}(d \eta)^{2}+h_{\zeta}(d \zeta)^{2},
$$

where $h_{\eta}$ and $h_{\zeta}$ are the scale factors, which are given by

$$
h_{\zeta}=\left[\left(\frac{\partial x}{\partial \zeta}\right)^{2}+\left(\frac{\partial y}{\partial \zeta}\right)^{2}\right]^{\frac{1}{2}}, \quad h_{\eta}=\left[\left(\frac{\partial x}{\partial \eta}\right)^{2}+\left(\frac{\partial y}{\partial \eta}\right)^{2}\right]^{\frac{1}{2}} .
$$

For a conformal mapping, $x_{\zeta}=y_{\eta}$ and $x_{\eta}=-y_{\zeta}$. Thus

$$
h_{\zeta}=\left[x_{\zeta} y_{\eta}-x_{\eta} y_{\zeta}\right]^{\frac{1}{2}}=\mathscr{J}_{\frac{1}{\frac{1}{2}}}, \quad h_{\eta}=\mathcal{J}_{\frac{1}{2}} .
$$

Writing the expression for the gradient as

$$
\nabla f=\frac{1}{h_{\zeta}} \frac{\partial f}{\partial \zeta} \mathbf{e}_{\zeta}+\frac{1}{h_{\eta}} \frac{\partial f}{\partial \eta} \mathbf{e}_{\eta}+\frac{1}{h_{z}} \frac{\partial f}{\partial z} \mathbf{e}_{z}
$$

substituting for $h_{\zeta}$ and $h_{\eta}$ and letting $f=\tilde{\psi}$ and $g=z$, the velocity for a two-dimensional flow parallel to the $\zeta, \eta$ plane is

$$
\mathbf{V}=\tilde{u} \mathbf{e}_{\zeta}+\tilde{v} \mathbf{e}_{\eta}=\left|\begin{array}{ccc}
\mathbf{e}_{\zeta} & \mathbf{e}_{\eta} & \mathbf{e}_{z} \\
\boldsymbol{J}_{-\frac{1}{2} \partial \tilde{\psi} / \partial \zeta} & \boldsymbol{J}-\frac{1}{2} \partial \psi / \partial \eta & \partial \psi / \partial z \\
0 & 0 & 1
\end{array}\right| .
$$

Thus $\tilde{u}=\tilde{J}-\frac{1}{2} \partial \psi / \partial \eta$ and $\tilde{v}=-J-\frac{1}{2} \partial \psi / \partial \zeta$. The velocity components can be written in terms of $J(x, y)$ by use of the identity

$$
\left[\begin{array}{ll}
\zeta_{x} & \zeta_{y} \\
\eta_{x} & \eta_{y}
\end{array}\right]\left[\begin{array}{ll}
x_{\zeta} & x_{\eta} \\
y_{\xi} & y_{\eta}
\end{array}\right]=\left[\begin{array}{ll}
1 & 0 \\
0 & 1
\end{array}\right]
$$

i.e.

which implies that $J=J^{-1}$. Thus

$$
[J][\tilde{J}]=[I]
$$

$$
\tilde{u}=J \frac{1}{2} \partial \psi / \partial \eta, \quad \tilde{v}=-J^{\frac{1}{2}} \partial \psi / \partial \eta
$$

\section{REFERENCES}

Burns, J. C. \& Parkes, T. 1967 Peristaltic motion. J. Fluid Mech. 29, 731-743.

Fung, Y. C. \& YIr, C. S. 1968 Peristaltic transport. J. Appl. Mech. 35, 669-675.

Hanin, M. 1968 The flow through a channel due to transversally oscillating walls. Israel $J$. Tech. $6(1-2), 67-71$.

JAFFrin, M. Y. \& Shaprro, A. H. 1973 Peristaltic pumping. Anr. Rev. Fluid Mech. 3, 13-36.

Lighthill, M. J. 1954 The response of laminar skin friction and heat transfer to fluctuations in the stream velocity. Proc. Roy. Soc. A 224, 1-23. 
Lrs, C. C. 1956 Motion in the boundary layer with a rapidly oscillating external flow. Proc. 9th Int. Cong. Appl. Mech. Brussels vol. 4, p. 155.

Schuichtivg, H. 1968 Boundary Layer Theory, 6th edn. McGraw-Hill.

STOKER, J. J. 1957 Water Waves. Wiley-Interscience.

Taylor, G. I. 1952 Analysis of the swimming of microscopic organisms. Proc. Roy. Soc. A 209, 447-461.

Wrrson, D. E. 1976 An investigation of peristaltic pumping with wind energy applications. Ph.D. dissertation, University of Texas, Austin.

Wrlson, D. E. et al. 1977 Peristaltic pumping by a lateral bending wave. Proc. A.S.M.E. Bioengng Symp., Atlanta, Georgia.

Yin, F. \& Fune, Y. C. 1969 Peristaltic waves in circular cylindrical tubes. J. Appl. Mech. 36, 579-587.

ZIEN, R. F. \& OstrACH, S. 1970 A long wave approximation to peristaltic motion. J. Biomech. $3,63-75$. 